\title{
Um Modelo para Trilhas de Aprendizagem em um Ambiente Virtual de Aprendizagem
}

\author{
David Brito Ramos ${ }^{1,2}$, Ilmara Monteverde Martins Ramos ${ }^{1,2}$, Priscilla Batista \\ do Nascimento ${ }^{1}$, Geovana de Souza Amaral ${ }^{1}$, Elaine Harada Teixeira de \\ Oliveira $^{1}$
}

${ }^{1}$ Instituto de Computação - Universidade Federal do Amazonas (UFAM) - Manaus AM - Brasil

${ }^{2}$ Instituto Federal de Educação, Ciência e Tecnologia do Amazonas (IFAM) - Campus Parintins - Parintins - AM - Brasil

\{davidness, ilmaramonteverde, priscilla.batista18,
gsamaral05\} @gmail.com, elaine@icomp.ufam.edu.br

\begin{abstract}
This paper aims to present a new model of learning paths, based on the interaction of students with learning management systems, which can be used as a learner model. Data were collected from closed classes and from the analysis performed, a representation of the model was designed as a directed graph. The resources and activities were represented as vertices, and the student's navigation (path) between the vertices as edges. The results of the experiments show that it is possible to use the proposed model to perform actions such as prediction of school dropout, group formation and behavior analysis.
\end{abstract}

Resumo. Este trabalho tem o objetivo de apresentar um novo modelo de trilhas de aprendizagem, baseado em relações de interação aluno-ambiente virtual de aprendizagem, que possa ser utilizado como modelo de aprendiz. Foram coletados dados de turmas já encerradas e a partir das análises realizadas, foi construida a representação do modelo no formato de grafo dirigido, onde os recursos e atividades foram representados como vértices, e a navegação (caminho) do aluno entre os vértices como arestas. Os resultados dos experimentos mostram que é possível utilizar o modelo proposto para realizar ações como predição de reprovação escolar, formação de grupos e análise de comportamento.

\section{Introdução}

O uso da Internet popularizou a Educação a Distância (EaD) como uma prática para se oferecer cursos regulamentados nas mais diversas áreas. Dados recentes disponibilizados pela Associação Brasileira de Educação a Distância (ABED) mostram o aumento no número de alunos registrados [ABED 2016]. Tal modalidade possui características e comportamentos que merecem atenção por parte do docente. Assim, um dos grandes desafios que surgiu com a $\mathrm{EaD}$ foi como identificar o comportamento dos alunos e/ou realizar o acompanhamento de suas atividades.

Nesse sentido, as pesquisas buscam apresentar ferramentas educacionais que considerem os dados referentes aos alunos como ponto importante na busca pelo ensino 
adaptado. Essas informações são chamadas de modelo do usuário (user model) ou modelo do aprendiz (student model). De acordo com Huang, Guerra-Hollstein e Brusilovsky [2016], um modelo de aluno representa o estado de conhecimento de domínio de um aluno. Assim com base no modelo do usuário, são executados uma série de algoritmos que buscam, em geral, recomendar o melhor recurso ou a melhor atividade a ser realizada pelo aluno. Na maioria dos trabalhos, esses recursos/atividades são analisados e classificados por especialistas que indicam fatores como nível de dificuldade, tempo esperado de execução, carga cognitiva exigida, entre outros.

Como visto acima, o modelo de ensino personalizado baseia-se em: dados do usuário, algoritmos de recomendação e recursos/atividades catalogados. Este trabalho busca contribuir com os demais por meio de um modelo de trilhas de aprendizagem (TAs), percursos realizados pelo aprendiz durante o seu processo de aprendizagem, no contexto dos Ambientes Virtuais de Aprendizagem (AVAs).

Diante deste cenário, este trabalho tem o objetivo de apresentar, baseado em relações de interação aluno-AVA registrados em um banco de dados, um novo modelo de trilhas de aprendizagem que possa ser utilizado como modelo de aprendiz. Diferente de trabalhos como de Gomes e Pimentel [2013] ou Ferreira, Barbosa e Gluz [2013], as informações sobre o usuário são coletadas de forma transparente.

As próximas seções apresentam os trabalhos relacionados (Seção 2), o processo metodológico para a criação do modelo e dos experimentos (Seção 3), o modelo de trilhas de aprendizagem proposto (Seção 4), as ferramentas desenvolvidas (Seção 5), os experimentos realizados (Seção 6), os resultados do trabalho (Seção 7), e por fim, a Seção 8 apresenta a conclusão.

\section{Trabalhos relacionados}

Com a visualização das TAs, como em Adesina e Molloy [2011], Teutsch e Bourdet [2010], Schröck et al. [2010] e Cerezo et al. [2014], o professor pode ter uma visão geral e simplificada de sua turma. Os trabalhos de Sitthisak, Gilbert e Albert [2013], Pires e Cota [2010], Vantroys e Peter [2003], Wisuttikul e Boonmee [2004] e Marquez et al. [2008] mostram que é possível utilizar informações sobre as TAs para a realização de ajustes nos AVAs. Assim, ao analisar as trilhas de aprendizagem, o professor pode compreender o comportamento dos alunos [Barnes 2005], verificar suas contribuições no AVA, conforme Teutsch e Bourdet [2010], e o seu processo de aprendizagem [Cerezo et al. 2014]. As TAs representam de forma natural o comportamento do aluno, pois conforme Ramos et al. [2015] e Gutiérrez, Pardo e Kloos [2006], elas são criadas sem interferências diretas do professor. O trabalho de Wisuttikul e Boonmee [2004] apresenta um modelo de recomendação de conteúdo (objetos de aprendizagem) predefinido. Nesse caso, a trilha de aprendizagem é considerada sob o ponto de vista do professor.

Neste trabalho, o termo 'trilhas de aprendizagem' refere-se ao percurso que o aluno faz ao interagir com os recursos e atividades do AVA. Ramos e Oliveira [2015] e Ramos et al. [2015] identificaram que a principal forma de representação de TAs são os grafos, modelagem esta escolhida para a pesquisa, pois permite ao mesmo tempo, a criação de um modelo teórico que armazena adequadamente as informações sobre TAs a representação dessas informações de forma clara. Este trabalho difere dos demais na forma que constrói e armazena as trilhas de aprendizagem, pois o modelo pode 
VI Congresso Brasileiro de Informática na Educação (CBIE 2017)

Anais do XXVIII Simpósio Brasileiro de Informática na Educação (SBIE 2017)

representar ao mesmo tempo a TA definida pelo professor e a gerada pelo aluno. Os dados necessários são coletados de forma transparente do próprio registro do AVA.

\section{Metodologia}

Foi realizada uma pesquisa bibliográfica por meio de uma revisão sistemática sobre TAs em AVAs, publicada em Ramos et al. [2015], e uma pesquisa de campo, onde foram coletados dados de um AVA, durante um semestre. Para isso, foram coletados dados de uma turma já encerrada, de um curso à distância de Informática Aplicada, com cinco turmas e mais de 100 alunos da Universidade Federal do Amazonas (UFAM), onde foram analisadas as interações registradas no banco de dados do AVA. A partir das análises realizadas, foi construída a representação do modelo no formato de grafo dirigido, onde os recursos e atividades foram representados como vértices, e a navegação (caminho) do aluno entre os vértices como arestas. Os vértices são ponderados com o número de interações, e as arestas possuem como peso o número de vezes que foram percorridas pelo aluno.

Em seguida, ferramentas foram desenvolvidas para o Moodle na forma de plugin, para analisar o modelo produzido. Os dados sobre as trilhas de aprendizagem foram representados de forma visual, e também em formato arff. Este último foi analisado utilizando-se alguns dos algoritmos de agrupamento e classificação disponíveis no Weka [Witten e Frank 2005], ferramenta de mineração de dados e aprendizagem de máquina. Assim, as trilhas de aprendizagem foram analisadas utilizando a teoria dos grafos, técnicas de mineração de dados e aprendizagem de máquina para a geração de modelos de comportamento. Posteriormente, foi realizada uma análise em duas disciplinas da UFAM: Introdução à Ciência dos Computadores e a disciplina de Matemática Discreta.

\section{Arquitetura do Trabalho}

As trilhas de aprendizagem são formadas a partir do histórico de acesso do aluno, que fica armazenado em banco de dados. Para esta pesquisa, utilizou-se o Moodle, popular AVA, que também é utilizado na universidade onde se realizou esta pesquisa. O Moodle mantém registros de histórico que indicam quais recursos e atividades foram acessados pelos alunos e quando foram acessados. Dessa forma, é possível ordenar cronologicamente os eventos ocorridos durante a interação do aluno com o AVA. Uma vez ordenados, os registros apresentam a sequência de navegação do aluno pelos recursos e atividades do ambiente. A partir da observação dessa estrutura e dos estudos realizados anteriormente, em Ramos e Oliveira [2015], concluiu-se que era possível a utilização da estrutura de grafos para a representação das trilhas de aprendizagem no Moodle.

O modelo de trilhas de aprendizagem deste trabalho utiliza o grafo dirigido como forma de representação. Os recursos e atividades do AVA foram representados como vértices, e a navegação (caminho) do aluno entre os vértices como arestas. Essa navegação é obtida por meio da sequência ordenada do registro do ambiente virtual. A seguir são dados mais detalhes sobre cada um deles.

Vértices representam os recursos e atividades fornecidos dentro do curso no AVA. Eles armazenam as seguintes informações: Ordem, número que indica a ordem do vértice na sequência definida pelo docente para recursos e atividades, ou seja, a trilha 
de aprendizagem estabelecida pelo professor; Nome do recurso/atividade, indica o nome dado pelo docente; Tipo do recurso/atividade, indica o tipo do recurso ou atividade como, por exemplo, 'Fórum' ou 'Tarefa'; Tópico, indica a qual tópico (unidade) o vértice pertence e; Número de interações (V), indica o total das interações (visualização, envio de arquivo, postagem, entre outras ações), sendo utilizado para ponderar o vértice.

As arestas representam a navegação (caminho) do aluno entre os vértices. Elas informam o número de vezes que ele parte de um vértice para outro, que é utilizado como peso da aresta. Sua origem e destino também são registrados. Além disso, as arestas foram classificadas em três tipos, dados $i$ e $j$ a ordem do vértice: aresta de avanço, indica uma navegação de um recurso/atividade para o outro mais à frente que o sucessor imediato, ou seja, dado um vértice $i$ e um vértice $j$, a aresta que parte de $i$ e incide em $j$ é do tipo avanço se $j>i+1$; aresta padrão, representa a navegação de um recurso/atividade para o sucessor imediato, ou seja, dado um vértice $i$ e um vértice $j$, a aresta que parte de $i$ e incide em $j$ é do tipo padrão se $j=i+1$ e; aresta de retorno, indica uma navegação de um recurso/atividade para outro anterior, nesse caso, dado um vértice $i$ e um vértice $j$, a aresta que parte de $i$ e incide em $j$ é do tipo retorno se $j<i$.

Com essas informações são calculadas a proporção da quantidade de cada tipo de aresta em relação ao total de arestas, e também a medida de dispersão, que indica o quão dispersa é a navegação do aluno. A medida de dispersão (disp) é dada pela média de comprimento das arestas. O comprimento de uma aresta é dado por $\operatorname{comp} p_{x}=j-i$, onde $i$ e $\boldsymbol{j}$ são a ordem dos vértices ligados pela aresta $\boldsymbol{x}$, e $\boldsymbol{n}$ é o número total de arestas. A medida de dispersão pode ser calculada considerando-se todas as arestas do grafo, ou por tipo de aresta.

$$
\operatorname{disp}=\frac{\sum_{x=1}^{n} \operatorname{comp}_{x}}{n}
$$

A Figura 1 apresenta o modelo de trilha de aprendizagem concebido. Nele, cada recurso/atividade possui uma cor e o diâmetro de cada vértice é proporcional ao número de interações $(\mathrm{V})$. Os valores nas arestas indicam o número de vezes que o aluno percorreu de um nó A para um nó B e a seta indica o sentido dessa transição. As arestas verdes indicam a ordem da TA estabelecida pelo professor, já as arestas azuis indicam que o aluno avançou para uma próxima tarefa diretamente, e por fim, as arestas vermelhas indicam que um aluno retornou para um recurso ou atividade anterior.

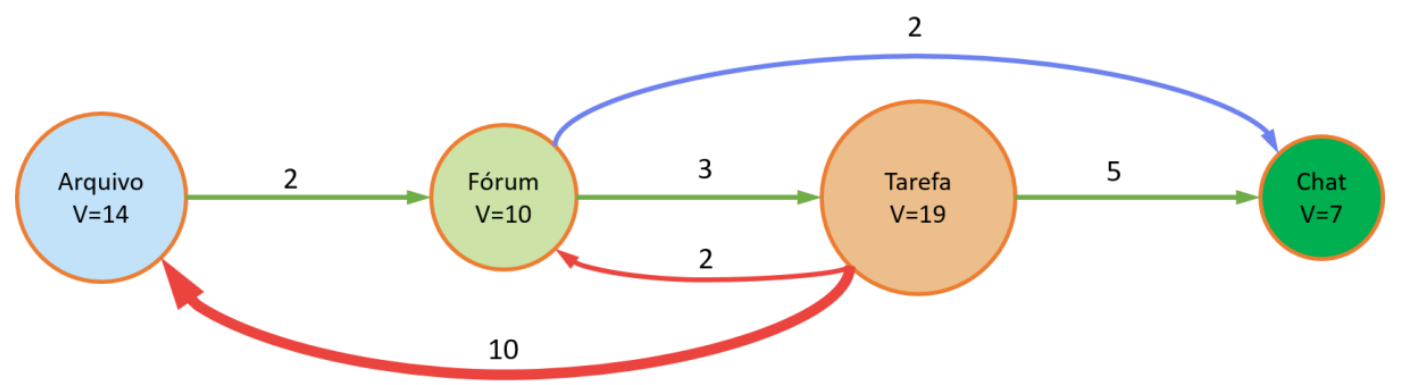

Figura 1. Modelo de trilha de aprendizagem 
Como o modelo baseia-se nos dados gerados e armazenados pelo AVA, é possível acompanhar a evolução da TA do aluno ao longo do tempo, pois o modelo pode utilizar dados apenas de um intervalo de tempo determinado pelo professor. $\mathrm{O}$ modelo também pode representar as TAs de uma turma, de forma geral; durante o decorrer das aulas, em tempo real; e também de uma turma já encerrada.

\section{Ferramentas criadas com o modelo}

Foram criadas ferramentas a partir do modelo de trilhas de aprendizagem proposto. $\mathrm{O}$ objetivo foi validar o modelo e experimentar algumas de suas possíveis aplicações.

A primeira ferramenta, chamada Learning Path Graph (LPGraph), de Ramos et al. [2016], gera o modelo de TAs e as armazena em dois formatos: JSON e arff. O formato JSON armazena os dados do modelo, que em seguida é renderizado por um script escrito com o uso de D3.js (biblioteca JavaScript) para criar uma representação da TA (Experimento 3). As TAs das Figuras 2, 3 e 4 foram geradas pelo LPGraph. Já o formato de arquivo arff permite que os dados das trilhas sejam manipulados pela ferramenta Weka. O arquivo arff, para este trabalho, armazena, para cada aluno ou a turma com um todo, uma lista de atributos tais como: identificação do aluno/turma; quantidade de acesso de cada vértices e total; percentual de cada tipo de aresta (avanço, padrão, retorno); dados de dispersão, entre outros.

A segunda ferramenta criada foi chamada de Moodle Cluster. Ela utiliza os dados do modelo em conjunto com o algoritmo K-Means para sugerir agrupamentos para atividades colaborativas.

\section{Experimentos}

Os experimentos foram realizados com a participação de alunos de duas disciplinas que utilizam o Moodle: Introdução à Ciência dos Computadores (ICC), com uma turma do curso de Física (28 alunos) e outra de Engenharia Elétrica (42 alunos), e a disciplina de Matemática Discreta (MD) (43 alunos) em uma turma de Ciência da Computação. Os cursos (disciplinas) utilizaram o formato de tópicos, com o conteúdo a ser tratado na ordem estabelecida pelos próprios docentes. Os recursos e atividades utilizados foram: Fórum, Arquivo, Página, Questionário (Quiz), Tarefa e URL. Cada tópico continha um fórum de dúvidas referente ao seu conteúdo. O recurso Arquivo foi utilizado para fornecer o conteúdo da aula, lista de exercícios e orientações para atividades práticas. $\mathrm{O}$ recurso Página e também o URL serviram para fornecer links para recursos externos ao Moodle. Já o Questionário e a Tarefa são atividades utilizadas para a avaliação dos alunos.

A Figura 2 apresenta uma visão parcial da TA da turma de Engenharia Elétrica, a Figura 3, da TA da turma de Física e a Figura 4 da TA da turma de Matemática Discreta. Para todas as turmas, as figuras mostram apenas as arestas mais usadas das trilhas. Os vértices são representados por círculos coloridos: a cor da borda indica o tópico ao qual ele pertence; a cor interna indica o tipo de recurso/atividade e está relacionada com as cores dos ícones do Moodle. As arestas, também coloridas, informam o número de vezes que um aprendiz parte de um vértice para outro, a espessura da aresta é proporcional a este valor. 
VI Congresso Brasileiro de Informática na Educação (CBIE 2017)

Anais do XXVIII Simpósio Brasileiro de Informática na Educação (SBIE 2017)

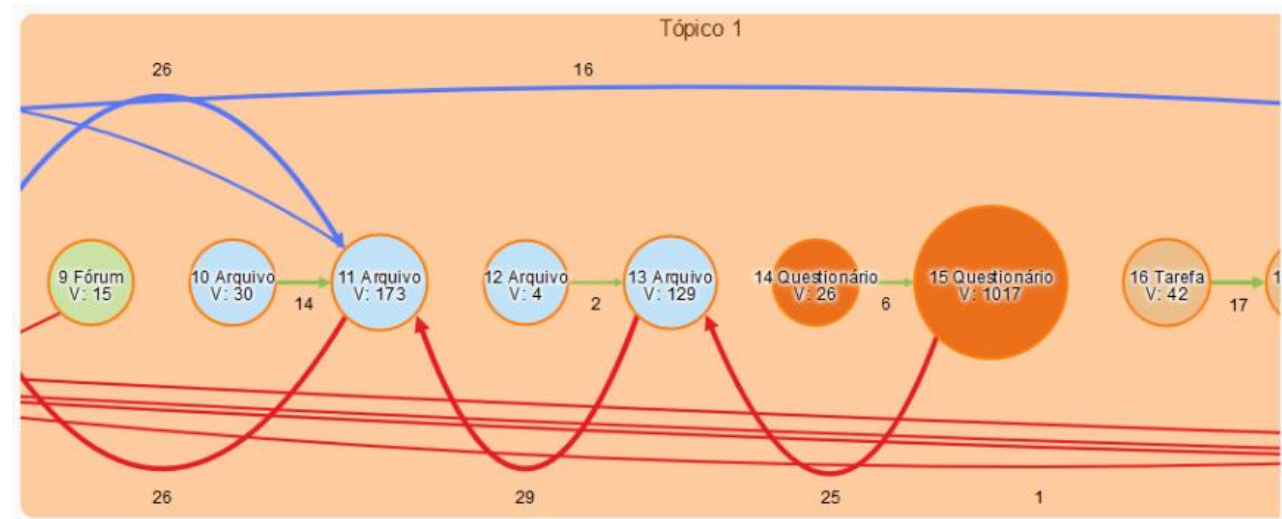

Figura 2 - Parte da TA da turma de Engenharia Elétrica na disciplina de ICC

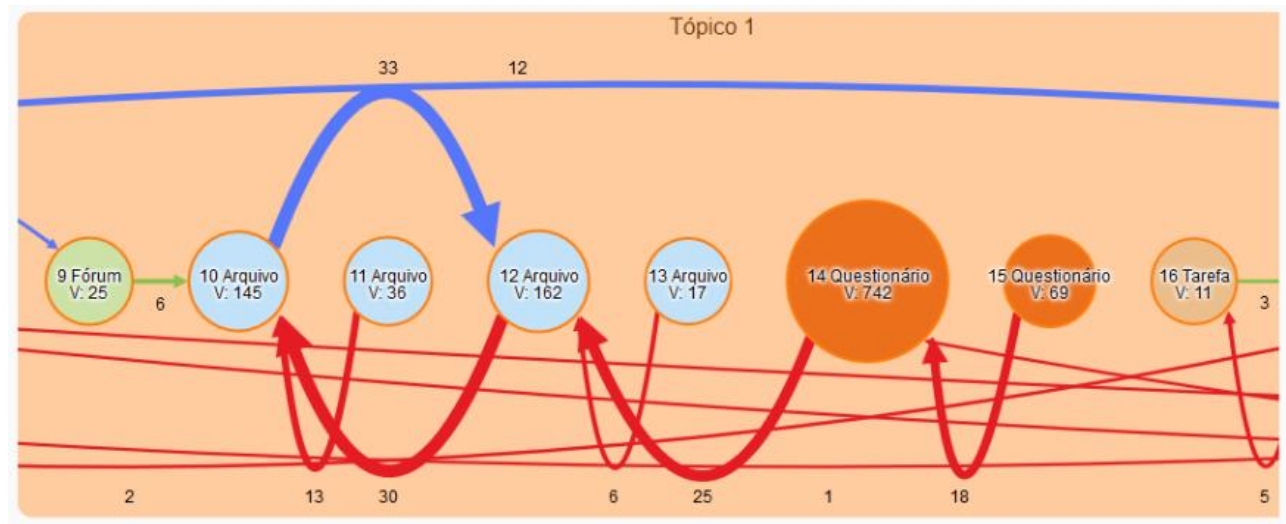

Figura 3 - Parte da TA da turma de Física na disciplina de ICC

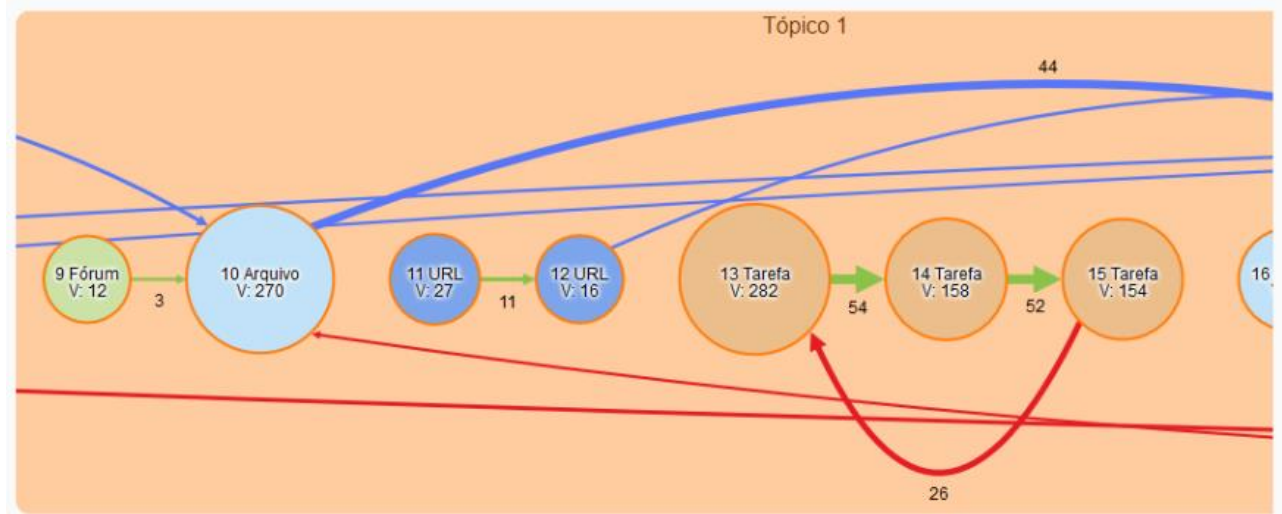

Figura 4 - Parte da TA da turma de Ciência da Computação na disciplina de MD

\subsection{Experimento 1: predição de reprovação}

Este experimento teve como objetivo verificar se o modelo poderia ser utilizado para prever a aprovação ou reprovação de um aluno. Para isso, foram analisados os dados das turmas quando elas já haviam encerrado. De posse dos dados das trilhas, juntamente com a condição de 'aprovado' ou 'reprovado' dos alunos, gerou-se um arquivo arff para cada turma. Os resultados foram obtidos por meio dos classificadores do Weka. Para os experimentos não se aplicou a engenharia de requisitos para seleção ou normalização dos atributos, sendo utilizados dados brutos. Para todas as turmas, foram avaliados 0 
VI Congresso Brasileiro de Informática na Educação (CBIE 2017)

Anais do XXVIII Simpósio Brasileiro de Informática na Educação (SBIE 2017)

desempenho dos classificadores: Naive Bayes, SVM, KNN (IB1, 1 vizinho), kNN (IB10, 10 vizinhos), e C4.5 (J48). A seguir, são apresentados os resultados de desempenho de cada um deles.

Para a turma de Engenharia Elétrica, todos os 42 alunos foram aprovados, segundo as notas do Moodle, dessa forma todos os classificadores tiveram um acerto de $100 \%$, exceto o Naive Bayes que classificou uma instância incorretamente, ficando com 97.6\% de acerto. Para a turma de Física, o maior valor de acerto foi de $96.4 \%$ para todos os classificadores, exceto o SVM que acertou somente $92.8 \%$. Para a turma de Matemática Discreta o maior valor de acerto foi para os classificadores Naive Bayes e C4.5 com 72.1\% de instâncias classificadas corretamente. O IB10 chegou a 69.7 \% de acertos, os demais tiveram desempenho inferior.

\subsection{Experimento 2: Formação de grupos}

O objetivo deste experimento foi verificar se o modelo proposto neste trabalho poderia ser utilizado para sugerir agrupamentos para atividades colaborativas. Foi utilizado o plugin chamado Moodle Cluster (M-Cluster). O M-Cluster é um mecanismo que dá ênfase à formação de grupos aplicando o algoritmo $K$-Means, que é utilizado com três métricas de similaridade, que são as distâncias: Euclidiana, Manhattan e Cosseno. Os atributos de cada aluno obtidos por meio dos dados extraídos do modelo foram: média de acesso de vértices, quantidade, dispersão e variância das arestas padrão, avanço e de retorno. O M-Cluster gera duas representações de sugestões de formação de grupos, uma descritiva e outra visual por meio de um gráfico de bolhas. Os agrupamentos são formados de acordo com a similaridade das trilhas, assim, considerando-as como modelo do estudante são gerados agrupamentos homogêneos. A ferramenta sugere ao docente três resultados de formação de grupos e o mesmo pode escolher qual é a mais adequada para os seus alunos em determinada atividade.

Um exemplo de utilização da ferramenta foi em uma turma de Matemática Discreta do ano de 2016/2 do curso de Ciência da Computação da UFAM na modalidade semipresencial. No experimento, o M-Cluster usou os atributos e classificou os alunos com o K-Means, obtendo três resultados de agrupamentos, um para cada métrica.

Esses grupos sugeridos pela ferramenta foram validados e visualizados pelo docente. Para validar os grupos sugeridos, foram realizadas duas atividades na disciplina. Na primeira atividade, os estudantes escolheram seus parceiros de grupo e na segunda, os grupos foram formados de acordo com as sugestões da ferramenta. Os experimentos mostraram que, de acordo com o docente especialista, a ferramenta obteve resultados satisfatórios onde $75 \%$ dos alunos igualaram ou melhoraram as notas em relação às notas alcançadas na primeira atividade. Foi constatado também, que do total de grupos formados, $30 \%$ foram grupos idênticos à primeira formação (os mesmos obtiveram bons resultados nas duas atividades). Esses resultados ainda não são conclusivos, mas já são um ponto de partida para análises futuras mais profundas.

\subsection{Experimento 3: Análise de comportamento}

Para a análise de comportamento, analisou-se visualmente as TAs. As Figuras 2 e 3 são da mesma disciplina, com os mesmos recursos e atividades, durante o mesmo intervalo de tempo, porém cada turma apresenta um comportamento diferente. A turma de Física 
VI Congresso Brasileiro de Informática na Educação (CBIE 2017)

Anais do XXVIII Simpósio Brasileiro de Informática na Educação (SBIE 2017)

tem uma navegação mais concentrada, o que pode ser observado pelas arestas mais largas.

Apesar da turma do curso de Física ser menor, o número de interações com vértices do tipo 'arquivo' foi bem semelhante ao número de interações do curso de Engenharia Elétrica. Ao final, a média da turma, para a atividade do questionário, foi levemente superior para a turma de Física, que no geral interagiu mais, se for considerado o número de alunos, são 28 alunos contra 42 da turma de Engenharia Elétrica. Porém, na média final do Tópico 1, a menor média, na comparação entre as duas turmas, foi da turma de Física que retornou a recursos anteriores com mais frequência, o que é indicado pela aresta vermelha, em relação às arestas padrão e de avanço. Isso parece indicar que a turma teve dificuldades para compreender o assunto do tópico e, por isso, precisou recorrer, mais vezes, aos conteúdos anteriores. A tendência, de voltar a recursos anteriores, se mantém mais elevada no decorrer da disciplina para a turma de Física. Comparando as médias finais, ao final da disciplina, a maior média é do curso de Engenharia Elétrica. Dessa forma, verificou-se que, para as turmas analisadas, uma proporção maior do número de arestas vermelhas indica que há uma grande chance de a turma estar enfrentando dificuldades no aprendizado.

A Figura 4, apresenta uma visão parcial da TA da turma de Matemática Discreta. Observa-se que a turma costuma acessar os recursos diretamente, porém acessaram pouco os links externos (URL). No Tópico 1 foram 27 e 16 acessos ao recurso URL, apesar de a turma ter 43 alunos matriculados. Com isso, o professor consegue identificar se um recurso/atividade que ele disponibilizou está sendo utilizado pelos alunos e, de posse dessa informação, poderia avaliar o porquê de os alunos não utilizarem esses recursos de forma efetiva.

Também foi verificado o uso do LPGraph para a análise de trilhas de aprendizagem de um aluno individualmente. Neste caso, foram observadas as TAs de alguns alunos, que, para o conteúdo do Tópico 1, tiveram notas altas e baixas. Percebeuse que para o primeiro caso, as trilhas dos alunos continham poucas arestas e, portanto, eram visualmente mais limpas, além disso, as arestas mais utilizadas são predominantemente as arestas de avanço, de cor azul. Já ao analisar as TAs de alunos com notas baixas, observou-se que há mais arestas e elas estão mais dispersas, e em geral a quantidade de arestas de retorno (vermelhas) é maior. Em conjunto com essa informação, um número pequeno de arestas também pode indicar uma tendência à evasão, o que será objeto de pesquisa em trabalhos futuros.

\section{Resultados}

Os resultados mostram que é possível utilizar o modelo proposto para realizar ações como predição de evasão, reprovação, formação de grupos e análise de comportamento.

Os estudos realizados foram de caráter empírico. Além da análise das ações de turmas em tempo real, o modelo foi utilizado juntamente com a ferramenta Weka para poder associar o comportamento do aluno à predição de reprovação, alcançando mais de $70 \%$ de acertos. O modelo também foi aplicado na formação de grupos para atividades colaborativas do Moodle, e nesse caso, os agrupamentos sugeridos foram analisados pela professora da disciplina que avaliou positivamente os resultados. $\mathrm{O}$ comportamento do aluno e da turma pode ser acompanhado pela ferramenta visual desenvolvida a partir do modelo proposto. 
VI Congresso Brasileiro de Informática na Educação (CBIE 2017)

Anais do XXVIII Simpósio Brasileiro de Informática na Educação (SBIE 2017)

\section{Conclusão}

Este trabalho apresentou a aplicação de um modelo de representação de trilhas de aprendizagem na previsão de possível reprovação em cursos no Moodle, formação de grupos e análise de comportamento. Uma ferramenta, chamada LPGraph, que identifica e representa TAs dos alunos que utilizam o Moodle foi utilizada. Nela foi adicionada uma extensão que gera um arquivo arff a partir dos dados da ferramenta. Esse arquivo foi analisado por meio da ferramenta Weka. Outra ferramenta desenvolvida a partir do modelo foi o $M$-Cluster, que faz sugestões de agrupamentos aplicando o algoritmo $K$ Means ao modelo proposto.

O modelo apresentado pode ser utilizado para muitas aplicações além das aplicadas neste trabalho. Alguns exemplos são: acompanhar a evolução de um aluno ao longo do tempo, verificar a influência dos recursos disponibilizados com as taxas de aprovação e reprovação, verificar quais tipos de trilhas são mais propensas a melhorar o desempenho do aluno, e também contribuir para a criação de AVAs adaptativos. Este trabalho conclui, portanto, que é possível utilizar o modelo proposto como fonte de análise e mineração de dados.

\section{Referências}

ABED. (2016). Censo EAD $\mid A B E D$. (Associação Brasileira de Educação a Distância) Retrieved 06 20, 2017, from Associaçao Brasileira de Educação a Distância: http://www.abed.org.br/site/pt/midiateca/censo_ead/

Adesina, A., e Molloy, D. (2011). A Business Process Management Based Virtual Learning Environment: Customised Learning Paths. In: International Conference on Computer Supported Education (CSEDU) (pp. 365-368). Noordwijkerhout: SciTePress.

Barnes, T. (2005). The Q-matrix method: Mining student response data for knowledge. In: American Association for Artificial Intelligence 2005 Educational Data Mining Workshop.

Cerezo, R., Suarez, N., Carlos Núñez, J., e Sánchez-Santillán, M. (2014). EGraph tool: Graphing the learning process in LMSs. In: International Conference on Learning Analytics And Knowledge (pp. 273-274 ). Indianapolis: ACM.

Ferreira, L. G., Barbosa, J. L., e Gluz, J. C. (2013). Um Modelo de Recomendação Ubíqua de Conteúdo para Grupos de Aprendizes. In: Simpósio Brasileiro de Informática na Educação (SBIE 2013), 24. Campinas.

Gomes, E. H., e Pimentel, E. P. (2013). Personalização do E-Learning Baseado no Nível de Aquisição de Conhecimentos do Aprendiz. In: Simpósio Brasileiro de Informática na Educação (SBIE 2013), 24. Campinas.

Gutiérrez, S., Pardo, A., e Kloos, C. D. (2006). Finding a learning path: toward a swarm intelligence approach. In: Proceedings of the 5th IASTED International Conference on Web-based Education (pp. 94-99). Puerto Vallarta: ACTA Press. Retrieved from http://dl.acm.org/citation.cfm?id=1166728.1166745

Huang, Y., Guerra-Hollstein, J. D., e Brusilovsky, P. (2016). Modeling skill combination patterns for deeper knowledge tracing. CEUR Workshop Proceedings. 1618. CEUR-WS. 
VI Congresso Brasileiro de Informática na Educação (CBIE 2017)

Anais do XXVIII Simpósio Brasileiro de Informática na Educação (SBIE 2017)

Marquez, J., Ortega, J., Gonzalez-Abril, L., e Velasco, F. (2008). Creating adaptive learning paths using ant colony optimization and Bayesian networks. In: International Joint Conference on Neural Networks (IJCNN) (pp. 3834-3839). Hong Kong : IEEE.

Pires, J. M., e Cota, M. P. (2010). Evolutive mechanism for E-Learning platforms: A new approach for old methods. In: IEEE EDUCON Education Engineering (pp. 891894). Madrid, Spain: IEEE.

Ramos, D. B., e Oliveira, E. H. T. (2015). Relatório Técnico da Revisão Sistemática de Literatura sobre Trilhas de Aprendizagem em Ambientes Virtuais de Aprendizagem. Universidade Federal do Amazonas, Manaus. Fonte: http://mobmoodle.icomp.ufam.edu.br/projeto/?page_id=10

Ramos, D. B., Oliveira, E. H. T., Ramos, I. M. M., e Oliveira, K. M. (2015). Trilhas de Aprendizagem em Ambientes Virtuais de Ensino-aprendizagem: Uma Revisão Sistemática da Literatura. In: XXVI Simpósio Brasileiro de Informática na Educação, 26, (pp. 338-347). Maceió.

Ramos, D. B., Ramos, I. M. M., Nascimento, P. B. e Oliveira, E. H. T. (2016). Uma Ferramenta Baseada em Grafo para Identificação e Visualização de Trilhas de Aprendizagem. In: XXI Congreso Internacional de Informática Educativa, TISE 2016, v. 12. (p. 237-243). Santiago: Nuevas Ideas en Informática Educativa.

Schröck, J., Bargel, B., Roller, W., e Rauner, A. (2010). Learning maps usage of cartographic metaphors for orientation in e-learning courses. In: IADIS International Conference on e-Learning, Part of the IADIS Multi Conference on Computer Science and Information Systems (pp. 50-56). Freiburg: IADIS Press.

Sitthisak, O., Gilbert, L., e Albert, D. (2013). Learning in Moodle using CompetenceBased Knowledge Space Theory and IMS QTI. In: International Computer Science and Engineering Conference (ICSEC) (pp. 53-57). Nakorn Pathom, Thailand: IEEE.

Teutsch, P., e Bourdet, J.-F. (2010). How to see training paths in learning management systems? In: International Conference on Advanced Learning Technologies (ICALT), 2010 IEEE 10th.

Vantroys, T., e Peter, Y. (2003). COW, a Flexible Platform for the Enactment of Learning Scenarios. (J. Favela, e D. Decouchant, Eds.) Groupware: Design, Implementation, and Use (Lecture Notes in Computer Science), 2806, (pp. 168-182).

Witten, I. H., e Frank, E. (2005). Data mining: Practical machine learning tools and techniques. Morgan Kaufman.

Wisuttikul, T., e Boonmee, C. (2004). A Study of Coaching Assisted System using Learning Object Value Model. In: IEEE Region 10 Conference (TENCON 2004). pp. 294 - 297. Chiang Mai: IEEE. 